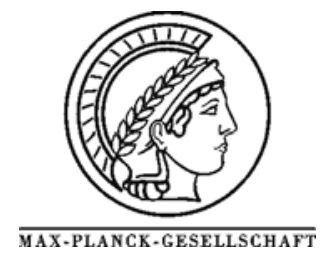

\title{
Ordered Arrays of Mesoporous Microrods from Recyclable Macroporous Silicon Templates
}

\author{
X. Chen ${ }^{1}$, M. Steinhart ${ }^{1}$, C. Hess ${ }^{2}$, U. Gösele ${ }^{1}$
}

\author{
${ }^{1}$ Max Planck Institute of Microstructure Physics, Weinberg 2, 06120 Halle/Saale, Germany \\ ${ }^{2}$ Department of Inorganic Chemistry, Fritz-Haber-Institute of the MPG, Faradayweg 4-6, 14195 Berlin, Germany \\ * Corresponding author: e-mail steinhart@mpi-halle.de,
}

Ordered mesoporous materials fabricated by exploiting self-assembled surfactants as molecular templates have been intensively investigated.[1-5] However, their integration into device architectures still remains a challenge. Recent efforts have focused on the synthesis of MCM (Mobil Crystalline Material)[6,7] and SBA (Santa Barbara amorphous) type[6,8-13] materials inside porous supports characterized by straight, aligned pores oriented normal to the surface. For this purpose, porous alumina matrices, either ordered[14] or disordered,[15] have predominantly been employed. The hybrid systems thus obtained are promising components for device architectures in the fields of catalysis and separation, where macroscopic membranes that consist of aligned pores with diameters of a few nanometers and high aspect ratios are required. The mesoporous rods can be released by a wet-chemical-etching step that destroys the alumina matrix. When the solvent of the suspension thus obtained is evaporated, capillary forces that act between the mesoporous rods result in the occurrence of large aggregates in otherwise disordered powders.

The preparation of ordered arrays of freestanding mesoporous microrods by means of recyclable macroporous silicon [16] templates is reported here. The microrods are removed from the template by a simple mechanical liftoff process or by the shrinkage of a macroscopic sol layer on the template surface upon calcination. Since no wetchemical-etching step is involved, condensation of the rods is avoided. It is assumed that such hierarchical systems, which combine features at the micrometer and nanometer scales, can be used in flow reactors, for the storage of lowmolar-mass species, for size-exclusion chromatography, as a sensor component, and as an array of artificial chaperones guiding the folding of proteins.[17] Macroporous Si is prepared by photoelectrochemical etching of lithographically prepatterned n-type silicon wafers.[16] The pores form either a hexagonal or quadratic monodomain that may extend several square centimeters and exhibits a sharp pore diameter distribution. The pore diameters can be adjusted to any value between $370 \mathrm{~nm}$ and several micrometers, and the pore depth is only limited by the thickness of the wafer used. 1D nano- and microstructures that consist of various materials have been fabricated using macroporous $\mathrm{Si}$ as a template,[18] by adapting a strategy initially introduced by Martin.[19]

The lateral arrangement of the mesoporous microrods that form inside the macroporous Si templates is determined by the lithographic prepatterning of the Si wafers into which the macropores are etched. Therefore, it should be possible to adjust the wetting properties of the microrod arrays by varying the size, shape, and lattice constants,[20] as well as to implement self-cleaning behavior.[21] An important aspect of the procedure reported here is that the mesoporous microrods are pulled out of the pores. The silicon template is therefore conserved and can be recycled. This is a prerequisite for upscaling this process and potentially enables the economic integration of mesoporous materials into device structures.

The macroporous $\mathrm{Si}$ is modified by a two-step procedure to minimize adhesion between the microrods and the pore walls. At first, a well-defined, smooth silica layer with a thickness of about $5 \mathrm{~nm}$ and a high density of hydroxyl groups is grown by treatment with a boiling $\mathrm{H}_{2} \mathrm{SO}_{4} / \mathrm{H}_{2} \mathrm{O}_{2}$ mixture. Subsequently, $1 \mathrm{H}, 1 \mathrm{H}, 2 \mathrm{H}, 2 \mathrm{H}$ perfluorodecyltrichlorosilane is grafted onto the pore walls 
by adapting protocols reported elsewhere[22] to render them into low-energy surfaces.[23] Mesoporous SBA-15 precursors are prepared following the procedures described in the literature, $[5,9,10]$ using a mixture that contains poly(ethylene oxide)-block-poly(propylene oxide)-blockpoly(ethylene oxide) triblock copolymer $\left(\mathrm{EO}_{20} \mathrm{PO}_{70} \mathrm{EO}_{20}\right)$ as a structure-directing agent, and tetraethyl orthosilicate (TEOS) as a silica source under acidic conditions (1.0M aqueous $\mathrm{HCl}$ ). The modified macroporous Si templates are covered with the SBA-15 precursor solutions thus prepared, and the SBA-15 sols are gelated at room temperature for two days and then at $60{ }^{\circ} \mathrm{C}$ for one day. After removal of the $\mathrm{EO}_{20} \mathrm{PO}_{70} \mathrm{EO}_{20}$ by calcination at $550{ }^{\circ} \mathrm{C}$ for $6 \mathrm{~h}$, mesoporous silica microrods connected to a silica film on the surface of the template are obtained inside the macroporous Si.

A simple and crude lift-off procedure is applied to pull the mesoporous rods out of the pores. The macroporous Si templates are turned upside down, and the silica film that covers the surface of the macroporous $\mathrm{Si}$ is glued onto a scanning electron microscopy (SEM) sample holder. The macroporous Si is then torn away with the aid of an adhesive tape. To recycle the templates, the silica layer that covers the pore walls, and the residual segments of the mesoporous microrods still located inside the pores, which break off during the lift-off, are etched with an aqueous solution of $5 \mathrm{wt} \% \mathrm{HF}$. The templates are then treated again with a boiling $\mathrm{H}_{2} \mathrm{SO}_{4} / \mathrm{H}_{2} \mathrm{O}_{2}$ mixture and modified with $1 H, 1 H, 2 H, 2 H$-perfluorodecyltrichlorosilane, as described above. The diameter of the template pores increases slightly by $20-30 \mathrm{~nm}$ during the course of this procedure.

The lift-off of the template, as it is performed here, imposes considerable shear forces on the rods. In order to explore up to which aspect ratio (length/diameter) the mesoporous microrods can be pulled out of the pores without breakage, templates with a pore diameter of $800 \mathrm{~nm}$ and a pore depth of $50 \mathrm{~lm}$ were used, which corresponds to an aspect ratio of $<60: 1$. Arrays of freestanding mesoporous microrods are obtained connected to a silica film that is initially located on the surface of the template and acts as a substrate after the lift-off of the macroporous Si. An example for this, where the mesoporous microrods have a length of about $15 \mathrm{~lm}$, is seen in Figure 1a. It is noted that the length of the rod segments that are pulled out of the pores varies for different areas of the samples, but is uniform over several hundreds of square micrometers. This indicates, on the one hand, that the strength of the occurring shear forces is not uniform over the entire sample. On the other hand, the position where failure occurs in the microrods is determined by the point of application of the transverse force rather than by intrinsic defects. This is obvious from Figure 1b, which shows a cross section of a macroporous Si template after lift-off. Inside the pores, segments of the mesoporous microrods with a uniform length of 7.5 Im are seen in the vicinity of the pore bottoms.
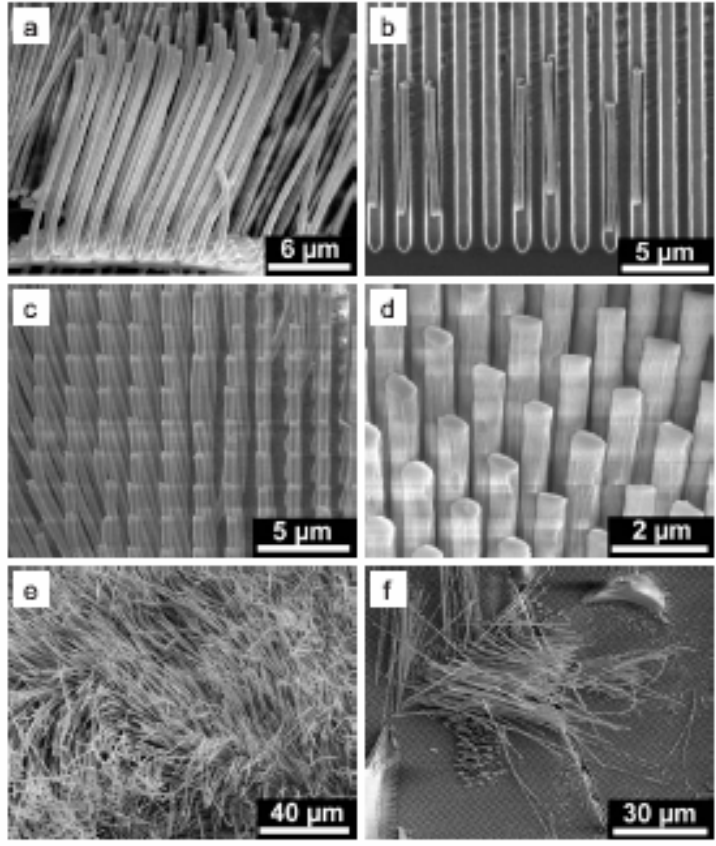

Figure 1: SEM images of mesoporous microrods pulled out of macroporous Si with a pore depth of $50 \mathrm{~lm}$. a) Cross-sectional view of some microrods attached to a silica film. b) Cross-sectional view of a cleaved macroporous silicon template after the lift-off of the mesoporous microrods. Inside the pores, rod segments with a uniform length of approximately $7.5 \mathrm{~lm}$ are seen, which were broken off during the lift-off. c,d) Ordered arrays of mesoporous microrods attached to an underlying silica film at different magnifications. e) Array of mesoporous microrods with high aspect ratios of the order of 50:1, attached to a silica film. f) Mesoporous microrods lying on the surface of a macroporous Si template after removal of the silica layer on the template surface by means of a scalpel.

Even without a specific setup that allows shear forces to be minimized, released rod segments with a length of 10-15 lm remain connected to the silica film, from which the macroporous $\mathrm{Si}$ is detached. The lateral arrangement of the rod segments corresponds to that of the pores in the templates used, that is, the order initially imposed by the template is conserved even after lift-off. Typical top views of mesoporous microrod at different magnifications are seen in Figure 1c and d. Because of the shrinkage upon calcination, the diameter of the mesoporous microrods of ca. $600 \mathrm{~nm}$ is smaller than that of the template pores (800 $\mathrm{nm}$ ). It is to be expected that the length of the mesoporous microrods is somewhat smaller than the depth of the template pores because of shrinkage upon calcination. Occasionally, rod segments with a length of several tens of micrometers are found (Fig. 1e). It is reasonable to assume that in this case, entire rods are pulled out of the pores without breakage. However, they do not form ordered arrays. Figure 1f shows mesoporous microrods lying on a macroporous Si template with a pore depth of $50 \mathrm{~lm}$, after the mechanical removal of the residual silica layer from the surface of the template by means of a scalpel. Their lengths 


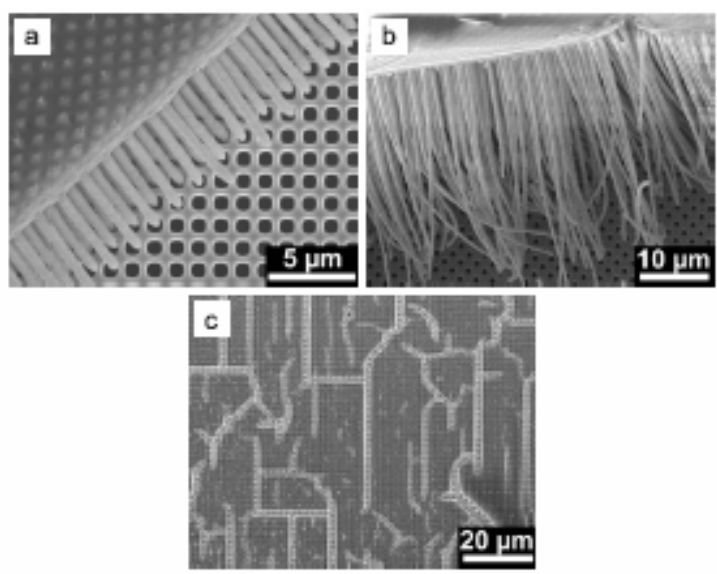

Figure 2: SEM images of mesoporous microrods connected with a silica surface film. a) Side view of a sample, for which macroporous Si with a pore diameter of $800 \mathrm{~nm}$ and a pore depth of $10 \mathrm{~lm}$ was used. The mesoporous microrods were partially pulled out of the pores because of shrinkage upon calcination. The thickness of the sol layer on the template surface during the gelation was about 5 $\mathrm{mm}$. b) Side view of mesoporous microrods connected to a silica film, which are partially embedded in macroporous $\mathrm{Si}$ with a pore diameter of $800 \mathrm{~nm}$, an interpore spacing of 1.3 $\mathrm{lm}$, and a pore depth of $100 \mathrm{~lm}$. Again, the thickness of the sol layer during the gelation was about $5 \mathrm{~mm}$. c) Top view of a silica film on macroporous Si prior to the lift-off of the mesoporous microrods.

amount to 30-40 lm, which indicates that the aspect ratio of the mesoporous microrods is only slightly smaller than that of the template pores, if they do not break during the liftoff.

If a $5 \mathrm{~mm}$ thick sol layer covers the templates with a perfluorated surface during the gelation step, an interesting phenomenon can be observed upon calcination. The mechanical stress associated with the shrinkage of the surface layer partially pulls the microrods out of the pores. A side view of an array of microrods connected with the silica surface film is shown in Figure 2a. The template used here has a pore diameter of $800 \mathrm{~nm}$ and a pore depth of $10 \mathrm{~lm}$. The distance between the silica film and the surface of the macroporous Si amounts to approximately $5 \mathrm{Im}$, which corresponds to the length of the freestanding rod segments. Only the lower segments of the microrods are still located inside the template pores. If the depth of the template pores is increased to $100 \mathrm{Im}$, even longer segments of the rods are pulled out of the pores, as seen in Figure 2b, where the freestanding rod segments have a length of about $35 \mathrm{~lm}$. The silica layer on the surface of the macroporous Si may act as a substrate for the mesoporous microrods after liftoff. Upon calcination, however, cracks form in this layer and separate intact areas that extend some hundreds of square micrometers (Fig. 2c). This drawback may be overcome by connecting an additional support layer to the silica surface layer prior to the lift-off.
The importance of the hydrophobic modification of the pore walls is obvious from a comparison between a sample prepared as described above, and another one where an untreated template is used. In the former case, the pores contain freestanding microrods that have no adhesive contact with the perfluorated pore walls. Figure 3a shows a top view of a sample thus treated, where the silica film on the template surface is removed with a sharp blade. A side view of the uppermost pore segments of a hydrophobized template that contains mesoporous microrods is seen in Figure 3b. In the latter case, the pore walls of the macroporous $\mathrm{Si}$ consist of a native silica layer. After hydrolysis of the TEOS and calcination, the material inside the pores is tightly attached to the pore walls, presumably because the silica formed by the hydrolysis of TEOS reacts with the hydroxyl groups of the pore walls (Fig. 3c). The material inside the pores adheres so strongly to the pore walls that shrinkage during calcination results in the generation of cracks and hollow spaces in the silica microrods rather than in a detachment from the pore walls.

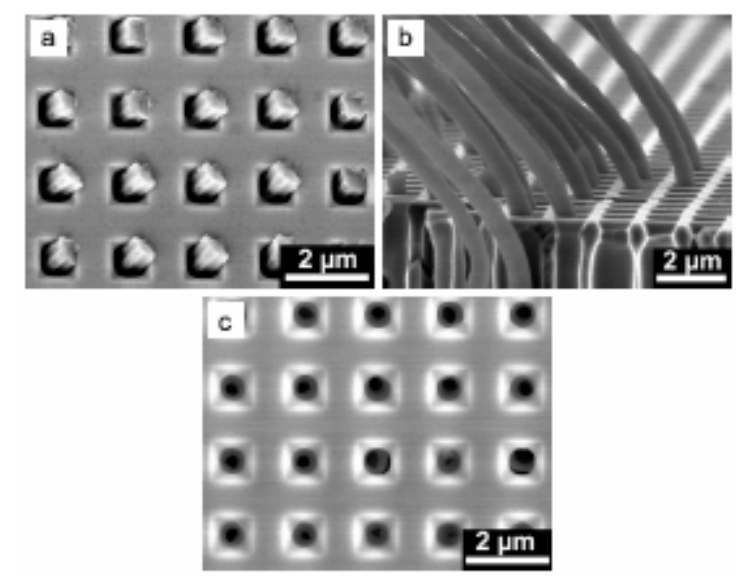

Figure 3: SEM images of mesoporous microrods inside macroporous Si. a) Top view and b) side view of macroporous $\mathrm{Si}$ with perfluorated walls that contain mesoporous microrods. c) Top view of macroporous Si with unmodified pore walls that contain mesoporous microrods.

The morphology of the mesoporous microrods is probed by transmission electron microscopy (TEM). The rods contain ordered arrays of mesopores with a diameter of about $6 \mathrm{~nm}$. In order to optimize their accessibility, the mesopores should be arranged perpendicular to the surface of the microrods and the rod axis, as seen in Figure 4a. The representative TEM image shows a microrod that has the desired mesopore orientation, which is indeed dominant under the conditions applied here and is found for approximately $70 \%$ of the investigated rod segments. In Figure $4 \mathrm{~b}$, an example is seen where the mesopores are aligned with the rod axis. This orientation is found for about $20 \%$ of the rod segments. Approximately $10 \%$ exhibit pores that are tilted with respect to the rod axis and the pore walls, as shown in Figure 4c. Whereas the pore orientation is uniform for rods 
with a length of $10 \mathrm{Im}$ or below, longer rods consist of segments with varying mesopore orientations. If required, the dominant orientation of the mesopores can easily be varied by changing the conditions of gelationand calcination, such as aging conditions and hydrolysis rates,[11,12] or the thermal history.[13] However, this should not affect the lift-off of the template and the accessibility of arrays of freestanding mesoporous microrods. It is demonstrated that mesoporous silica microrods prepared inside macroporous silicon templates with perfluorated pore walls can easily be pulled out of the pores. Even though a crude lift-off procedure is applied, ordered arrays of mesoporous microrods with aspect ratios of the order of 15 can easily be obtained. It is assumed that the shear force can be significantly reduced by the use of advanced setups, allowing the coherent and reliable removal of macroscopic ensembles of microrods from the templates. This is a general prerequisite for the up-scaling of template-based approaches for the fabrication of one-dimensional nanostructures and microstructures.

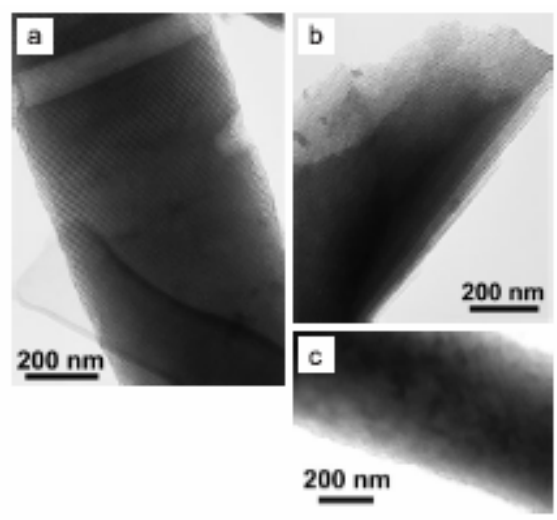

Figure 4: TEM images of released mesoporous microrods. a) Mesoporous microrod that has the mesopores perpendicular to the surface and the rod axis. This orientation was dominant in the investigated ensemble of rods. b) Mesoporous microrod that has the mesopores arranged parallel with the surface and the rod axis. c) Mesoporous microrod that has the pores tilted with respect to the surface and the long axis.

\section{References and Notes}

[1] C. T. Kresge, M. E. Leonowicz, W. J. Roth, J. C. Vartuli, J. S. Beck, Nature 1992, 359, 710.

[2] G. S. Attard, J. C. Clyde, C. G. Göltner, Nature 1995, 378, 366.

[3] Y. F. Lu, R. Ganguli, C. A. Drewien, M. T. Anderson, C. J. Brinker, W. L. Gong, Y. X. Guo, H. Soyez, B. Dunn, M. H. Huang, J. I. Zink, Nature 1997, 389, 364.

[4] M. Templin, A. Franck, A. Du Chesne, H. Leist, Y. Zhang, R. Ulrich,V. Schädler, U. Wiesner, Science 1997, 278, 1795.

\section{Experimental}

Macroporous silicon templates were prepared according to procedures described elsewhere [16] and then treated with a boiling mixture containing $98 \% \mathrm{H}_{2} \mathrm{SO}_{4}$ and $30 \% \mathrm{H}_{2} \mathrm{O}_{2}$ at a volume ratio of 7:3 for 20 min (caution: strong oxide). $1 H, 1 H, 2 H, 2 H$-perfluorodecyltrichlorosilane was then grafted onto the pore walls as follows: The macroporous silicon templates were rinsed with deionized water and dried in a nitrogen flow. Subsequently, the templates were heated at $85{ }^{\circ} \mathrm{C}$ for $2 \mathrm{~h}$ and at $130{ }^{\circ} \mathrm{C}$ for $3 \mathrm{~h}$, respectively, in the presence of $0.05 \mathrm{~mL}$ of $1 \mathrm{H}, 1 \mathrm{H}, 2 \mathrm{H}, 2 \mathrm{H}$ perfluorodecyltrichlorosilane (97\%, ABCR GmbH). Mesoporous SBA-15 precursor solutions were prepared according to protocols reported in the literature [2, 9, 10]. A typical procedure involves mixing TEOS (99+ \%, Alfa Aesar), $\mathrm{EO}_{20} \mathrm{PO}_{70} \mathrm{EO}_{20}$ (BASF, weight-average molecular weight $M \mathrm{w}=5800 \mathrm{~g} \mathrm{~mol}-1$, Pluronic P123), $1.0 \mathrm{M}$ $\mathrm{HCl}(\mathrm{aq})$ and ethanol as described elsewhere $[9,10]$. The macroporous Si templates were immersed in the precursor solution, and gelated at room temperature for $48 \mathrm{~h}$ and then at $60{ }^{\circ} \mathrm{C}$ for $24 \mathrm{~h}$. Finally, the samples were calcinated at $550{ }^{\circ} \mathrm{C}$ for $6 \mathrm{~h}$. SEM investigations were performed using a JEOL JSM 6300 operated at an accelerating voltage of 5 $\mathrm{kV}$. For TEM investigations, the mesoporous microrods were released by sonification of the templates in ethanol. The suspension thus obtained was then dropped onto copper grids coated with a perforated carbon film. The measurements were performed with a JEOL 1010 transmission electron microscope operated at $100 \mathrm{kV}$.

\section{Acknowledgement}

This work was supported by the Volkswagen Foundation (Thematic Impetus "Interplay between Molecular Conformations and Biological Function”, Az. I/80 780). Christian Hess thanks the Deutsche Forschungsgemeinschaft (DFG) for providing an Emmy Noether fellowship. We thank Kornelia Sklarek, Dr. Sven Matthias, and Andreas Langner for the preparation of macroporous silicon.
[5] D. Y. Zhao, J. Feng, Q. Huo, N. Melosh, G. H. Fredrickson, B. F. Chmelka, G. D. Stucky, Science 1998, 279, 549.

[6] G. Kickelbick, Small 2005, 1, 168.

[7] A. Yamaguchi, F. Uejo, T. Yoda, T. Uchida, Y. Tanamura, T. Yamashita, N. Teramae, Nat. Mater. 2004, 3, 337.

[8] Z. Yang, Z. Niu, X. Cao, Z. Yang, Y. Lu, Z. Hu, C. C. Han, Angew. Chem. Int. Ed. 2003, 42, 4201.

[9] Q. Lu, F. Gao, S. Komarneni, T. E. Mallouk, J. Am. Chem. Soc. 2004, 126, 8650. 
[10] Y. Wu, G. Cheng, K. Katsov, S. W. Sides, J. Wang, J. Tang, G. H. Fredrickson,M. Moskovits, G. D. Stucky, Nat. Mater. 2004, 3, 816.

[11] B. Yao, D. Fleming, M. A. Morris, S. E. Lawrence, Chem. Mater. 2004, 16, 4851.

[12] K. Jin, B. Yao, N. Wang, Chem. Phys. Lett. 2005, 409, 172.

[13] W. Zhu, Y. Han, L. An, Microporous Mesopororous Mater. 2005, 84, 69.

[14] a) H. Masuda, K. Fukuda, Science 1995, 268, 1466. b) H. Masuda, M. Satoh, Jpn. J. Appl. Phys., Part 2 1996, 35, 126. c) H. Masuda, F. Hasegawa; S. Ono, J. Electrochem. Soc. 1997, 144, L127. d) H. Masuda, K. Yada, A. Osaka, Jpn. J. Appl. Phys., Part 2 1998, 37, 1340. e) K. Nielsch, J. Choi, K. Schwirn, R. B. Wehrspohn, U. Gösele, Nano Lett. 2002, 2, 677.

[15] For example, Whatman Anodisc.

[16] a) V. Lehmann, J. Electrochem. Soc. 1993, 140, 2836. b) A. Birner, U. Grüning, S. Ottow, A. Schneider, F. Müller, V. Lehmann, H. Föll, U. Gösele, Phys. Status Solidi A 1998, 165, 111. c) A. Birner, R. B. Wehrspohn, U. M. Gösele, K. Busch, Adv. Mater. 2001, 13, 377.

[17] a) A. Brinker, G. Pfeifer, M. J. Kerner, D. J. Naylor, F. U. Hartl, M. Hayer-Hartl, Cell 2001, 107, 223. b) F. Takagi, N. Koga, S. Takada, Proc. Natl. Acad. Sci. USA 2003, 100, 11 367.
[18] a) M. Steinhart, J. H. Wendorff, A. Greiner, R. B. Wehrspohn, K. Nielsch, J. Schilling, J. Choi, U. Gösele, Science 2002, 296, 1997. b) M. Steinhart, R. B. Wehrspohn, J. H. Wendorff, ChemPhysChem 2003, 4, 1171. c) M. Steinhart, R. B. Wehrspohn, U. Gösele, J. H. Wendorff, Angew. Chem. Int. Ed. 2004, 43, 1334. d) Y. Luo, S. K. Lee, H. Hofmeister, M. Steinhart, U. Gösele, Nano Lett. 2004, 4, 143. e) P. Göring, E. Pippel, H. Hofmeister, R. B. Wehrspohn, M. Steinhart, U. Gösele, Nano Lett. 2004, 4, 1121. f) L. L. Zhao, M. Steinhart, M. Yosef, S. K. Lee, T. Geppert, E. Pippel, R. Scholz, U. Gösele, S. Schlecht, Chem. Mater. 2005, 17, 3. g) L. L. Zhao, M. Yosef, M. Steinhart, P. Göring, H. Hofmeister, U. Gösele, S. Schlecht, Angew. Chem. Int. Ed. 2006, 45, 311. h) L. L. Zhao, T. H. Lu, M. Zacharias,J. Yu, J. Shen, H. Hofmeister, M. Steinhart, U. Gösele, Adv. Mater. 2006, 18, 363.

[19] C. R. Martin, Science 1994, 266, 1961.

[20] D. Öner, T. J. McCarthy, Langmuir 2000, 16, 7777.

[21] W. Barthlott, C. Neinhuis, Planta 1997, 202, 1.

[22] a) S. R. Wasserman, Y. Tao, G. M. Whitesides, Langmuir 1989, 5, 1074. b) H. Tada, H. Nagayama, Langmuir 1995, 11, 136. c) A. Y. Fadeev, T. J. McCarthy, Langmuir 2000, 16, 7268.

[23] H. W. Fox, E. F. Hare, W. A. Zisman, J. Phys. Chem. 1955, 59, 1097. 